\title{
Introduction to fermionic structures
}

\author{
in $C^{4}$ space-time
}

\section{D.Mastoridis - 1}

Amaliados 17, Athens, Greece, P.O.Box 11523, d.mastoridis@prv.ypeka.gr

$$
\text { K.Kalogirou - } 2
$$

Amaliados 17, Athens, Greece, P.O.Box 11523, k.kalogirou@prv.ypeka.gr

keywords:unified theories;geometric unification;fermionic geometry;Cartan's principle of triality;complex space-time 


\begin{abstract}
We explore several ways, in order to include fermionic structures naturally in a physical theory in $C^{4}$. We begin with the standard Dirac formalism and we proceed by using Cartan's property of triality as a second option. Afterwards, we suggest a new approach (in a preliminary basis), by introducing an 1-linear form, as the "square root of the geometry" derived by the usual 2-linear forms (quadratic forms). Keeping this way, we introduce n-linear forms, in order to formulate a new geometric structure, which could be suitable for the formulation of a pure geometric unified theory.
\end{abstract}




\section{Introduction}

In the papers [?] [?] [?] [?] we have identified, that only bosons have appeared in our consideration and that they were appeared or constructed by the usual geometry of quadratic forms, leaving no room to introduce fermions by the geometry itself. But in general, in the usual context of physics, spinors can be defined and introduced mathematically by the geometric algebra, which is actually the Clifford algebra of a defined vector space equipped with a quadratic form. The most famous example is the Dirac spinor denoted as $\psi$ which is the solution of Dirac equation. In this paper we want to investigate several ways to introduce fermions within our consideration. We would like also to add a crucial comment of M. Atiyah, which show us the difficult situation that spinors have put us.

"No one fully understands spinors. Their algebra is formally understood but their general significance is mysterious. In some sense they describe the "square root" of geometry and, just as understanding the square root of -1 took centuries, the same might be true of spinors"

\section{Dirac's approach}

Let us start by exploring Dirac's approach by the beginning. In the Minkowski space we have the elementary length

$$
d s^{2}=n_{\mu \nu} d x^{\mu} d x^{\nu}
$$

where $n_{i j}$ is the Minkowski metric tensor. The action

$$
S=m c \int d s
$$

lead us to the geodesic and Hamilton-Jacobi equations. From Hamilton-Jacobi equation we derive the energy-momentum 4 -vector

$$
P=\left(\frac{E}{c}, p\right)
$$

and the squared $\mathrm{P}$ give us the energy-momentum relation

$$
E^{2}=p^{2} c^{2}+m^{2} c^{4}
$$


Afterwards, we pass to quantum physics by introducing the operators $\widehat{p}$ and $\widehat{E}$, which lead us to the Klein-Gordon equation in natural units

$$
\left(n^{\mu \nu} \partial_{\mu} \partial_{\nu}-m^{2}\right) \varphi=0
$$

At this point, everything started by an elementary length defined by a quadratic form in the flat Minkowski space and we were led to bosons. This is mainly the "road" or way of thinking that we have used in our consideration, in order to derive a bosonic equation in the curved $C^{4}$ space, as he wave seen in the third paper [?]. Afterwards, Dirac managed to derive the square root of the Hamiltonian by introducing a new Hamiltonian of first order derivatives in time and space as

$$
H=\alpha p+\beta m
$$

where $\alpha^{2}=\beta^{2}=1$ and $\{\alpha, \beta\}=0$ and finally has given us the Dirac equation

$$
i \gamma^{\mu} \partial_{\mu} \psi-m \psi=0
$$

and the Dirac matrices satisfy the algebra formed by

$$
\left\{\gamma^{\mu}, \gamma^{\nu}\right\}=n^{\mu \nu} I_{4}
$$

As a natural step, we can repeat the whole procedure if we start by our Klein-Gordon type equation in the flat $C^{4}$ or $R^{8}$ given by the Lagrangian as it was illustrated in the third paper

$$
L=\frac{\partial \mathcal{S}}{\partial x^{\mu}} \frac{\partial \mathcal{S}^{*}}{\partial x_{\mu}}-\frac{\partial \mathcal{S}}{\partial y^{\mu}} \frac{\partial \mathcal{S}^{*}}{\partial y_{\mu}}
$$

or by introducing, as we have seen, $\mathcal{S}=\varphi$ in natural units

$$
L=\frac{\partial \varphi}{\partial x^{\mu}} \frac{\partial \varphi^{*}}{\partial x_{\mu}}-\frac{\partial \varphi}{\partial y^{\mu}} \frac{\partial \varphi^{*}}{\partial y_{\mu}}
$$

This way, a Dirac equation type will be

$$
i \Gamma^{\mu} \partial_{\mu}^{c} \Psi=0
$$


where $\partial_{\mu}^{c}$ is the Cauchy derivative and $\Gamma^{\mu}$ are the Gell-Mann matrices in the flat space $R^{8}$ given by the relation

$$
\left\{\Gamma^{\mu}, \Gamma^{\nu}\right\}=n^{\mu \nu} I_{8}
$$

and $n^{\mu \nu}$ is the flat metric tensor in $R^{8}$ with signature $(4,4)$. The only left is to identify the type of the spinor $\Psi$ which accompanies this signature. In general, every spinor in any space is defined by its signature. As a consequence we must give the following informations [?] [?] $[?]$

1. in an even $\mathrm{d}=\mathrm{p}+\mathrm{q}$ dimensions metric tensor

$n^{\mu v}=\operatorname{diag}(++++,----)$ gamma matrices $\Gamma^{\mu}$ satisfies the Clifford algebra

$\Gamma^{\mu} \Gamma^{\nu}+\Gamma^{\nu} \Gamma^{\psi}=2 n^{\mu \nu}$

2. In $p-q=0 \bmod 8$ we have a Clifford algebra $C(4,4)$ and because $p+q=2 \times 4$ we have a real $2^{4}=16$ representation.

3. For $p-q=0 \bmod 8, p+q=0 \bmod 8$ we have a unique irreducible representation which is a real Majoran-Weyl one.

4. The M-W spinors satisfy both of the following conditions:

$\gamma^{(d+1)} \psi=\psi, \bar{\psi}=\psi^{+} C_{ \pm}$

and exist only if $p-q=0 \bmod 8$ for us $d=p+q=8$

5. If $d=p+q=0 \bmod 8$ we have only kinetic terms in the Lagrangian of the form $K_{x y}$.

Where $K_{x y}$ :

$$
K_{x y}=\Psi_{R}^{T} C \Gamma^{\mu} \partial_{\mu} \Psi_{L}+\lambda \Psi_{L}^{T} C \Gamma^{\mu} \partial_{\mu} \Psi_{R}
$$

6. For the $(4,4)$ signature the $\left(4_{s}+4_{A}\right)$ - representation of $\Gamma$ matrices has to be employed for both values on $n= \pm 1$ in order to provide a M-W basis.

7. The gamma matrices are given by $(3)$

$$
\begin{gathered}
\Gamma^{9}=\left(\begin{array}{cc}
1_{8} & 0 \\
0 & -1_{8}
\end{array}\right), \\
\Gamma^{i}=\left(\begin{array}{cc}
0 & \sigma^{c} \\
\bar{\sigma}^{c} & 0
\end{array}\right) i=1 \ldots 8
\end{gathered}
$$

(as presented in appendix 2 in [?])

Where $\bar{\sigma}_{i}=-\bar{\sigma}_{i}^{T}, i=1 \ldots 4$ (antisymmetric) 
$\bar{\sigma}_{i}=\bar{\sigma}_{i}^{T}, i=5,6,7,8$ (symmetric) and the diagonal charge-conjucation matrices are given by:

$$
\begin{aligned}
& C^{-1}=1_{4} \oplus-1_{4} \\
& \bar{C}^{-1}=-n C^{-1}
\end{aligned}
$$

the terms in the Lagrangian will have the general form

$$
i \bar{\Psi} C \Gamma^{\mu} \widehat{P_{\mu}} \Psi=i \bar{\Psi} C \Gamma^{\mu}\left(\left(\widehat{p_{\mu}}\right)_{g}+i\left(\widehat{p_{\mu}}\right)_{I}\right) \Psi
$$

and solving to $p_{g}$, in the case of flat space

$$
i \bar{\Psi} C \Gamma^{\mu} \frac{\partial}{\partial z^{\mu}} \Psi=i \bar{\Psi} C \Gamma^{\mu}\left(\frac{\partial}{\partial x^{\mu}} \Psi+i \frac{\partial}{\partial y^{\mu}}\right) \Psi
$$

and if

$$
\Gamma^{\mu} \frac{\partial}{\partial y^{\mu}} \Psi=m \Psi
$$

we will have the usual Dirac term

$$
i \bar{\Psi} \gamma^{\mu} \frac{\partial}{\partial y^{\mu}} \Psi-m \bar{\Psi} \Psi=0
$$

The question that must be answered is how the original M-W representation, breaks after embedding. In order to get a straight-full answer, we should study extensively the broken symmetry groups as they were presented in [?]. But even if we manage such a task, there will remain two big problems

1. In the case of bosons, we have started by a $d s^{2}$, we have found the geodesic equation, we have formed the H-J equation and after the second extremisation, we have identified the action $\mathcal{S}$ as the field. But, in Dirac approach we cannot identify the original definition of the spinor $\Psi$ as we have done with $\varphi$. We do not have a same procedure for fermions, similar to the case of bosons. The only thing left is to guess that

$$
\mathcal{S}_{f}=\sqrt{\hbar} \Psi
$$


In our opinion we should seek a better more geometrically way

2. The second problem, is related with the proper definition of a unified Lagrangian. In standard model we have three different Lagrangians, $L_{f}$ for fermions, $L_{b}$ for bosons and $L_{i n t}$ for interactions of the fields $F_{\mu \nu} F^{\mu \nu}, W_{\mu \nu} W^{\mu \nu}, G_{\mu \nu} G^{\mu \nu}$ forming a unified Lagrangian as

$$
L=L_{f}+L_{b}+L_{i n t}
$$

plus the Higg's mechanism. The question is how we can add these Lagrangians mathematically or geometrically, in the sense of a bigger geometry that can describe the unified Lagrangian. There should exist some geometric form d, where the Lagrangian of this form $L(d)$, should break as Eq. (18.).

In the two following paragraphs, we will try to investigate and propose some answers in a preliminary base.

\section{Cartan's property of triality}

We have seen in the first paper, about Cartan's property or principle of triality, concerning a triality that the three signatures $(4,4),(8,0),(0,8)$ share. We will refer to this, as signature triality. But, there is another one triality that exists, as it was illustrated in [?] [?] [?], that we will refer as spinor-vector triality.

"Let us conclude that Triality can be seen not only as a source of duality-mappings, but as an invariance property. In the original Cartan's formulation this is seen as follows. At first, a group $\mathrm{G}$ of invariance is introduced as the group of linear homogeneous transformations acting on the $8 \times 3=24$ dimensional space, leaving invariant, separately, the bilinears $B_{V}, B_{S^{+}}, B_{S^{-}}$ for vectors, chiral and antichiral spinors respectively (the spinors are assumed commuting in this case) plus a trilinear term T. Next, the Triality group $G_{T r}$ is defined by relaxing one condition as the group of linear homogeneous transformations leaving invariant $\mathrm{T}$ and the total bilinear $B_{\text {sum }}$ :

$$
B_{\text {sum }}=B_{V}+B_{S^{+}}+B_{S^{-}}
$$

it can be proven that $G_{t r}$ is given by the semidirect product of $\mathrm{G}$ and $S_{3}$

$$
G_{t r}=G \otimes S_{3}
$$

Let us consider $V=R^{8} \equiv C^{4}$ then the signatures $(4,4) \leftrightarrow(0,8) \leftrightarrow(8,0)$ concludes a Majorana-Weyl representation for $S^{+}, S^{-}$. Moreover, $S^{+}, S^{-}$are necessary 8 dimensional real spaces. As a result $B_{V}, B_{S^{+}}, B_{S^{-}}$are each one invariant under $S O(8)$ creating the product $S O(8) \times S O(8) \times S O(8)$ for the $B_{\text {sum }}$. Consequently, in our case $G=S O(8)$ and the group that leaves invariant $\mathrm{T}$ is $S O(8) \otimes S_{3}$ or $\operatorname{Spin}(8)$. Let us consider as p the number of plus(+) in signature, $\mathrm{q}$ the number of minus(-) and $\mathrm{d}$ the dimension of the space 


$$
(p, q)=(4,4) \quad d=p+q=8
$$

Then $d=0 \bmod 8$ and $p-q=0 \bmod 8$. From $\mathrm{d}$ and $\mathrm{p}$-q we can conclude that we have a real 8 dimensional Majorana-Weyl representation for the spinor spaces and that the group of automorphisms is $S O(8)$. Let us consider $(V, G),\left(S^{+}, s\right),\left(S^{-}, s\right)$ where $V=C^{4} \equiv R^{8}, G$ the hermitian metric tensor $S^{+}, S^{-} 8$ dimensional spinor spaces and s spin invariant inner-product

$$
s=\overline{(\psi, \varphi)}=\left(\psi^{c}, \varphi^{c}\right) \quad \forall \varphi, \psi \in
$$

Moreover $(V, G),\left(S^{+}, s\right),\left(S^{-}, s\right)$ are isomorphical as orthogonal spaces and the Triality B ensures the isometry (because of $S_{3}$ ) between the spaces. Once again, s will be the chargeconjugation operator $\mathrm{C}$, which preserves the spinor spaces and it can be used to raise and low indices. In order to unify the scheme between $V, S^{+}, S^{-}$we could "bosonise" $S^{+}, S^{-}$or "fermionise" V

$$
\begin{gathered}
B_{V}=V_{m}^{T}\left(g^{-1}\right)^{m n} V_{n} \\
B_{S^{+}}=\Psi^{T} C^{-1} \Psi \\
B_{S^{-}}=X^{T} C^{-1} X \\
T_{T r}=\Psi^{T} C \Gamma^{m} \Psi=2\left(\Psi^{T} C^{-1} \sigma^{m} X V_{m}\right)
\end{gathered}
$$

where $\Psi=\left(\begin{array}{c}\Psi_{a} \\ X_{a}\end{array}\right), a=1, \ldots, 8$. This trilinear form can serve us, as the geometric form $\mathrm{d}$ whose Lagrangian $L(t r)$ is the unified one. Moreover, this trilinear form, breaks into the three above mentioned spaces, where each one can form as associated Lagrangian as, $L_{S^{+}}$, $L_{S^{-}}$and $L_{V}$. Thus, through Cartan's spinor-vector triality, we have a more consistent way to present the addition of $L_{F}$ and $L_{b}$. In addition, we have the original symmetry of the trilinear form, given by the group $\operatorname{Spin}(8)$. This way, we can propose an answer, concerning the second above mentioned question. But, the first one, still cannot be answered. 


\section{New geometric structure}

We will try, to propose a new way, in order to give some satisfactory answers in both questions, in a preliminary basis. We will take Atiyah's expression literally about the square root of the geometry. Let us start with an $R^{n}$ space in the beginning and an elementary length as

$$
d s^{2}=g_{i j} d x^{i} d x^{j}
$$

where $g_{i j}$ is a bilinear or 2-linear form, which is the usual symmetric metric tensor. We van also, define the length

$$
d s=\sqrt{g_{i j} d x^{i} d x^{j}}
$$

as it exists in the usual context of geometry. Furthermore, we will impose the following question; Can we define the elementary length $d s$ in a different way using a 1-linear form instead? Let us consider that there exists a 1-liner form $g_{i}$, so that the elementary length can be written as

$$
d s=g_{i} d x^{i}
$$

If this expression is valid, it must satisfy the relation

$$
d s * d s=\left(g_{i} d x^{i}\right) *\left(g_{j} d x^{j}\right) \equiv g_{i j} d x^{i} d x^{j}
$$

where $\left(^{*}\right)$ is a product that must be defined. But, it must also satisfy a second relation also as

$$
g_{i} d x^{i} \equiv \sqrt{g_{i j} d x^{i} d x^{j}}
$$

If this consideration could be proved, we would have the advantage to study a new geometric structure, provided by the 1-linear form. This way, we could define an action $S\left(g_{i}\right)$, which would driven us to the derivation of geodesic equation with respect to $g_{i}$. Afterwards, we could continue by expressing the associated Hamilton-Jacobi and finally by a second extremisation, we should be able to find a "Dirac type "equation in $R^{n}$. Now, let us consider, that all these can be accomplished(we will give some proposals about this task below) and define a new geometric quantity $f\left(d_{2}\right)$ as 


$$
d_{2}=d s+d s^{2}
$$

and equip the space $R^{n}$ by the geometry that $f\left(d_{2}\right)$ defines as

$$
\left(R^{n}, f\left(d_{2}\right)\right) \equiv\left(R^{n}, g_{i}, g_{i j}\right)
$$

This consideration, leads us to the combination of functional analysis and differential geometry. This combination could be nicely expanded by defining series of different equipped geometries as

$$
d_{n}=\sum a_{i} d s^{i}=a_{0}+a_{1} d s+a_{2} d s^{2}+\ldots .+a_{n} d s^{n}
$$

and equip $R^{n}$ with $f\left(d_{n}\right)$, where we can define n-linear forms as

$$
\begin{gathered}
d s=g_{i} d x^{i} \\
d s^{2}=g_{i j} d x^{i} d x^{j} \\
d s^{3}=g_{i j k} d x^{i} d x^{j} d x^{k} \\
\cdot \\
d s^{n}=g_{i j \ldots n} d x^{i} d x^{j} \ldots . . d x^{n}
\end{gathered}
$$

We will return now, in the case of $d s$. If

$$
d s^{2}=g_{i j} d x^{i} d x^{j}=\left(a_{i} d x^{i}\right)\left(b_{j} d x^{j}\right)
$$

it must satisfy $a * b=\left(a_{i} b_{j}\right)$. If $g_{i j}$ symmetric

$$
g_{i j}=a_{i} b_{j}=a_{j} b_{i} \longrightarrow a_{i} b_{j}-a_{j} b_{i}=0 \longrightarrow a * b=0
$$

But, then the matrix

$$
C=\left(\begin{array}{cc}
a_{1} & b_{1} \\
a_{2} & b_{2} \\
\cdot & \cdot \\
\cdot & \cdot \\
a_{n} & b_{n}
\end{array}\right)
$$


must be of rank 1, which means

$$
b=\lambda a \longrightarrow a_{i} b_{j}=\lambda a_{i} a_{j} \longrightarrow g_{i j}=a_{i} a_{j}
$$

But the relation $g_{i i}=a_{i}^{2}$ must hold, which means

$$
g_{i i}=a_{i}^{2} \longrightarrow a_{i}= \pm \sqrt{\left|g_{i i}\right|}
$$

but this way we will have

$$
g_{i j}=\left( \pm \sqrt{\left|g_{i i}\right|}\right)\left( \pm \sqrt{\left|g_{j j}\right|}\right)
$$

which are the extra conditions. In the case of a Hermitian space we have an elementary length

$$
d s^{2}=G_{i j} d z^{i} d \bar{z}^{j}=a_{i} \bar{b}_{j} d z^{i} d \bar{z}^{j}=\left(a_{i} d z^{i}\right)\left(\overline{b_{i} d z^{j}}\right)
$$

A natural generalisation leads us to the relation $a * b=\left(a_{i} \bar{b}_{j}\right)$. If, $G_{i j}=a_{i} \bar{b}_{j}$, then $G_{j i}=a_{j} \bar{b}_{i}$ and $\bar{G}_{j i}=\bar{a}_{j} b_{i}$ because it must be $\bar{G}_{j i}=G_{i j} \longrightarrow a_{i} \bar{b}_{j}=\bar{a}_{j} b_{i}$. If, we set

$$
b_{i}=\lambda a_{i} \longrightarrow \bar{\lambda} a_{i} \bar{a}_{j}-\lambda \bar{a}_{j} a_{i}=0 \longrightarrow(\bar{\lambda}-\lambda) a_{i} \bar{a}_{j}=0 \longrightarrow \lambda \in R
$$

but then $G_{i j}=\bar{\lambda} a_{i} \bar{a}_{j}$ or $G_{i j}=a_{i} \bar{a}_{j}$ and

$$
G_{j i}=a_{j} \bar{a}_{i} \longrightarrow \bar{G}_{j i}=\bar{a}_{j} a_{i}=G_{i j}
$$

which means that $G_{i j}$ is Hermitian as it should be and the consideration holds. As a consequence, we can write

$$
d s^{2}=\left(a_{i} d z^{i}\right)\left(\overline{a_{j} d z^{j}}\right)
$$

and it must be also be

$$
G_{i i}=a_{i} \bar{a}=\left|a_{i}\right|^{2} \longrightarrow\left|a_{i}\right|=\sqrt{G_{i i}}
$$


but because $G_{i j}=a_{i} \bar{a}_{j}$ we can have

$$
\left|G_{i j}\right|=\left|a_{i}\right|\left|\bar{a}_{j}\right|=\sqrt{G_{i i}} \sqrt{G_{j j}}
$$

which expresses the extra conditions. It seems that $G_{i j}$ is $\sqrt{G_{i i}} \sqrt{G_{j j}}$ multiplied by an amplitude $\varphi$ ! If we return in Eq. (34), this power series could be presented by a function $f(d s)$. For example, if this power series represents a geometric one, the elementary length of this geometry will be

$$
\frac{1}{1-d s}
$$

or if it is presented by an exponential function will be

$$
e^{d s}
$$

The next step is to define series of the form of Eq. (34) in complex spaces $C^{n}$. This way, we will meet our consideration of a physical theory in $C^{4}$. An interesting task, would be to investigate whereas the action

$$
S_{\pi}=\int Z \sqrt{G} d \Omega
$$

can be derived naturally, by an action presented by the defined geometry with elementary length of $d s^{4}$ where the variation should be with respect to the length and not with respect to the metric tensor. If the dimension of the space can be written as $n=2 k$, there will be k elementary physical objects defined by $d s, d s^{2}, \ldots d s^{k}$, while the rest will be composited objects. Accordingly, in $C^{4}$ there will be only two elementary objects, one presented by $d s$ (fermions, $f \bar{f}$ ) and the second presented by $d s^{2}$ (bosons, $b \bar{b}$ and $b \longrightarrow f \bar{f}$ ). The other powers will present composite objects. For instance, $d s^{3}$, will present the interaction of $b \bar{b} f \bar{f}$ and $d s^{4} b \bar{b} b \bar{b} \longrightarrow f \bar{f} f \bar{f} f \bar{f} f \bar{f}$ and we can work the same way with the other powers. But finally, the whole series could present by a function $f(d s)$, where all the interactions could at once presented by this function. This function eventually, could give us all the information needed.

\section{Conclusion}

This paper is totally different with the other four [?] [?] [?] [?] of this series. In this paper, we rather suggest a new approach or a programme, that could be helpful in order to establish a unified theory, or even better to understand how Cosmos can be described. Our main belief, is that we have to redirect our focus mathematically in the study of $C^{n}$ spaces. Afterwards, we should proceed with the study of $H^{n}$ and $O^{n}$ spaces, as well and finally to seek a unified scheme for $R^{n}, C^{n}, H^{n}, O^{n}$ spaces. The final proposed step, is to "lift" from ordinary geometric structures to what we have called $f(d)$ geometric structures. 


\section{References}

\section{References}

[1] M. A. D. Andrade, F. Toppan, Real Structures in Clifford Algebras and Majorana Conditions in Any Space-time, Mod. Phys. Lett. A14 (1999) 1797-1814.

[2] M. A. D. Andrade, M. Rojas, F. Toppan , The Signature Triality of Majorana-Weyl Spacetimes , Int. J. Mod. Phys. A16 (2001) 4453-4480.

[3] H. L. Carrion, M. Rojas, F. Toppan, Quaternionic and OctonionicSpinors, A Classification, JHEP 0304 (2003) 040.

[4] D. Mastoridis, K. Kalogirou, $C^{4}$ Space-Time.. a window to new Physics, www.researchgate.net.

[5] D. Mastoridis, K. Kalogirou, Field Equations in $C^{4}$ space-time, www.researchgate.net.

[6] D. Mastoridis, K. Kalogirou, Introduction to Quantum Field Theory in $C^{4}$ space-time, www.researchgate.net.

[7] D. Mastoridis, K. Kalogirou, Dynamic Path in $C^{4}$ space-time, www.researchgate.net. 